\title{
Glucose metabolism after pediatric cardiac surgery: Too many sweets will ruin your appetite
}

\author{
Tara Karamlou, MD, ${ }^{\mathrm{a}}$ and Peter Oishi, $\mathrm{MD}^{\mathrm{b}}$
}

\footnotetext{
From the a Division of Pediatric Cardiac Surgery, University of California, San Francisco, Benioff Children's Hospital, San Francisco, Calif; and ' ${ }^{\mathrm{D}}$ Department of Pediatrics, University of California, San Francisco, Benioff Children's Hospital, and Associate Investigator, Cardiovascular Research Institute, San Francisco, Calif. Disclosures: Authors have nothing to disclose with regard to commercial support.

Received for publication July 11, 2015; accepted for publication July 14, 2015; available ahead of print Aug 5, 2015.

Address for reprints: Tara Karamlou, MD, Division of Pediatric Cardiac Surgery, University of California, San Francisco, Benioff Children's Hospital, Mail Stop 0117, 550 16th St, 5th Floor, San Francisco, CA 94518 (E-mail: tara.karamlou@ucsf.edu).

J Thorac Cardiovasc Surg 2015;150:505-6

$0022-5223 / \$ 36.00$

Copyright (C) 2015 by The American Association for Thoracic Surgery

http://dx.doi.org/10.1016/j.jtcvs.2015.07.041
}

The study by Floh and colleagues ${ }^{1}$ in this issue of the Journal addresses the relationship between cardiopulmonary bypass (CPB)-induced inflammation and glucose metabolism. This is an intriguing and very important pathophysiologic interaction, but the clinical relevance for investigating these responses is to inform patient stratification and develop perioperative management protocols, such as glycemic control pathways, that could improve outcomes. Therefore, one critical question to consider is whether there is evidence that glycemic control pathways are actually beneficial in this population. The Surgical Care Improvement Project is responsible for defining many of the quality measures that are tracked in hospitals. These metrics are subsequently endorsed by the National Quality Forum and included in The Joint Commission's Specifications Manual for hospital quality core measures. One measure, known as Surgical Care Improvement Project Infection (INF)-4, tracks the percentage of "cardiac surgery patients with controlled postoperative blood glucose." To meet this quality target, a patient undergoing cardiac surgery must have a blood glucose less than or equal to $180 \mathrm{mg} / \mathrm{dL}$ "in the timeframe of 18 to 24 hours after anesthesia end time." 2 The rationale for this metric initially was based on extrapolated data from critically ill adults demonstrating an association between hyperglycemia and adverse outcomes, and then expeditiously cemented by data from Zerr and colleagues ${ }^{3}$ and Furnary and colleagues ${ }^{4}$ showing that hyperglycemia was associated with an increased prevalence of sternal wound infections and early mortality. However, more recent evidence has seriously questioned not only the soundness of tight glycemic control as a quality metric but also the safety of adhering to the metric in the adults after cardiac surgery. The wisdom of tight glycemic control among pediatric patients is even more controversial, because the recent Safe Pediatric Euglycemia After Cardiac Surgery trial demonstrated no reduction in infectious complications or mortality among infants up to 36 months

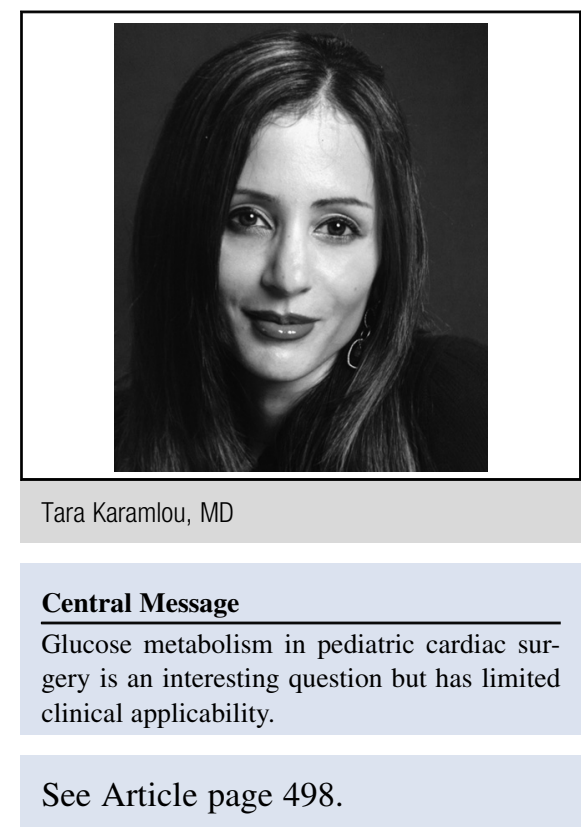

undergoing cardiac surgery. ${ }^{5}$ Moreover, multiple studies have shown increased morbidity and mortality among those in whom "tight glycemic control" was instituted, and these studies have led to the effective revocation of the current quality metric. ${ }^{6}$ Therefore, the clinical relevance of the study by Floh and colleagues ${ }^{1}$ is reduced despite the elucidation by the authors that the discrepant outcomes with aggressive glucose control may be related to the undoubtedly multifactorial underpinnings of stress hyperglycemia and their articulated notion that empiric patient selection may identify those most likely to benefit. If the latter is true, the next critical question to ask is, has this study provided useful data to inform patient selection regarding glucose management postoperatively? I would argue that it has not, predominantly because of the population chosen for the study. The authors performed a retrospective review of a prospectively collected dataset (from a prior randomized trial investigating the impact of ischemic preconditioning) of 299 children undergoing cardiac surgery involving CPB over a 3-year period at their institution. The authors' main hypothesis was that CPB-induced inflammation was associated with hyperinsulinemia and insulin resistance, and that these were associated with hyperglycemia. On the basis of their data, the authors conclude that higher cytokine values (with the exception of tumor necrosis factoralpha) were associated with hyperglycemia and higher insulin levels, but insulin resistance was not associated 
with higher cytokine levels. Overall, the concept is interesting, but truthfully, their study was a negative study, in that the glucose-insulin ratio was not decreased, and therefore may not be a major causative factor in inflammationmediated hyperglycemia after CPB in children. Frankly, I think that the population chosen for this study was one of convenience rather than one who should ideally have been chosen to investigate these relationships. The majority of the patients underwent simple atrial septal defect or ventricular septal defect repairs, and therefore are not the patients in whom CPB-mediated inflammation plays a critical role in clinical outcomes. Neonates undergoing complex repairs involving the use of hypothermia or prolonged exposure to CPB are the population who should be used. This sentiment was echoed recently in the study by Agus and colleagues, ${ }^{5}$ in which the authors focused on a high-risk subgroup of infants undergoing cardiac surgery in an effort to investigate whether the lack of evidence supporting tight glycemic control was due to heterogeneity of treatment effect. Furthermore, the intriguing study of metabolic profiling segregated by the aggressiveness of glycemic control by Correia and colleagues ${ }^{7}$ demonstrated that the strength of the metabolic response to surgery was influenced by the complexity of the congenital heart defect, with patients in Risk Adjustment in Congenital Heart Surgery-1 level 4 having a more profound impact than those in Risk Adjustment in Congenital Heart Surgery-1 level 2. Clearly, the authors of the present study would be ideally poised to investigate this question more deeply on the basis of their excellent prior work in this area. Additional investigations also may take the next step toward the elaboration of best practices based on prospective application of different glycemic control protocols.

Finally, the authors have failed to account for several important factors in their multivariable analysis, including the tem- perature, use of ultrafiltration, use of blood products, and lowest hematocrit on CPB. The use of older blood, the volume of blood, a lower hematocrit, and importantly, the temperature at which CPB is conducted are all important modulators of the systemic inflammatory response after CPB. Given the inherent complexities of glucose homeostasis and the inflammatory cascade, a complete dataset that includes all variables known to modulate these responses is critical.

The authors' approach of investigating the pathobiology to identify a subset of patients who might benefit from glycemic control is a good one; however, given the current ambiguity of the benefits of glycemic control in pediatric patients after cardiac surgery and the low-acuity patient population selected for this study, we are still a long way away from the stated goal.

\section{References}

1. Floh A, Manlhiot C, Redington A, McCrindle BW, Clarizia NA, Caldarone CA, et al. Insulin resistance and inflammation are a cause of hyperglycemia after pediatric cardiopulmonary bypass surgery. $J$ Thorac Cardiovasc Surg. 2015;150:498-504.e1.

2. LaPar DJ, Isbell JM, Kern JA, Ailawadi G, Kron IL. Surgical Care Improvement Project measure for postoperative glucose control should not be used as a measure of quality after cardiac surgery. J Thorac Cardiovasc Surg. 2014;147:1041-8.

3. Zerr KJ, Furnary AP, Grunkemeier GL, Bookin S, Kanhere V, Starr A. Glucose control lowers the risk of sternal wound infection in diabetics after open heart surgery. Ann Thorac Surg. 1997;63:356-61.

4. Furnary AP, Gao G, Grunkemeier GL, Wu Y, Zerr KJ, Bookin SO, et al. Continuous insulin infusion reduces mortality in patients with diabetes undergoing coronary artery bypass grafting. J Thorac Cardiovasc Surg. 2003;125: 1007-21.

5. Agus MS, Asaro LA, Steil GM, Alexander JL, Silverman M, Wypij D, et al. Tight glycemic control after pediatric cardiac surgery in high-risk patient populations. A secondary analysis of the Safe Pediatric Euglycemia After Cardiac Surgery Trial. Circulation. 2014;129:2297-304.

6. NICE-SUGAR Study Investigators, , Finfer S, Liu B, Chittock DR, Norton R, Myburgh JA, McArthur C, et al. Hypoglycemia and risk of death in critically ill patients. N Engl J Med. 2012;367:1108-18.

7. Correia GD, Ng KW, Wijeyesekera A, Gala-Peralta S, Williams R, MacCarthyMorrogh S, et al. Metabolic profiling of children undergoing surgery for congenital heart disease. Crit Care Med. 2015;43:1467-76. 